TITLE:

\title{
Mn-Mn couplings in Mn-doped CdS nanocrystals studied by magnetic circular dichroism spectroscopy
}

\section{$\operatorname{AUTHOR}(\mathrm{S}):$}

Taguchi, Seiji; Ishizumi, Atsushi; Tayagaki, Takeshi; Kanemitsu, Yoshihiko

\section{CITATION:}

Taguchi, Seiji ...[et al]. Mn-Mn couplings in Mn-doped CdS nanocrystals studied by magnetic circular dichroism spectroscopy. Applied Physics Letters 2009, 94(17): 173101.

\section{ISSUE DATE:}

2009-04

URL:

http://hdl.handle.net/2433/87343

\section{RIGHT:}

c 2009 American Institute of Physics. 


\title{
Mn-Mn couplings in Mn-doped CdS nanocrystals studied by magnetic circular dichroism spectroscopy
}

\author{
Seiji Taguchi, ${ }^{1}$ Atsushi Ishizumi, ${ }^{2}$ Takeshi Tayagaki, ${ }^{1}$ and Yoshihiko Kanemitsu ${ }^{1,3, a)}$ \\ ${ }^{1}$ Institute for Chemical Research, Kyoto University, Uji, Kyoto 611-0011, Japan \\ ${ }^{2}$ Graduate School of Materials Science, Nara Institute of Science and Technology, Ikoma, Nara 630-0192, \\ Japan \\ ${ }^{3}$ Photonics and Electronics Science and Engineering Center, Kyoto University, Kyoto 615-8510, Japan
}

(Received 4 March 2009; accepted 6 April 2009; published online 27 April 2009)

\begin{abstract}
We report on the optical and magnetic properties of Mn-doped CdS nanocrystals coated with a ZnS shell layer (CdS:Mn/ZnS core-shell nanocrystals) by magnetic circular dichroism (MCD) spectroscopy. The magnetic field and temperature dependences of the MCD spectrum show paramagnetic behavior of the CdS:Mn/ZnS core-shell nanocrystals. The MCD intensity increases with the Mn concentration up to a few mol \%, and then starts to decrease rapidly. This Mn-concentration dependence of the MCD intensity can be explained by the formation of Mn-Mn pairs in heavily doped nanocrystals. (C) 2009 American Institute of Physics.
\end{abstract}

[DOI: $10.1063 / 1.3125433]$

Over the past two decades, semiconductor nanocrystals (NCs) have been the subject of numerous studies due to interest both in the fundamental physics and potential applications in optoelectronic devices. ${ }^{1-3}$ Doped semiconductor NCs show unique multifunctional properties beyond those of undoped NCs. ${ }^{4,5}$ Transition-metal ions (e.g., Mn ions) are one of the most important and multifunctional dopants because these dopant ions act as luminescent centers and localized spins in semiconductor NCs. ${ }^{4,5}$ Mn-doped semiconductor NCs are model materials for the physics of impurity-doped semiconductors, and many efforts have been made for doping of $\mathrm{Mn}$ ions into semiconductor NCs. ${ }^{6-15}$

Several models have been proposed and argued for the impurity doping mechanism in semiconductor NCs. ${ }^{4}$ Studies have reported that the impurities are easily annealed out to the surface by the self-purification effect of the NCs, and thus solubility in NCs is much lower than in bulk crystals. ${ }^{8,9,13}$ However, the growth kinetic model shows that doping efficiency is determined by surface morphology, NC shape, and surfactants. Consequently, dopant solubility in NCs is higher or lower than that in bulk crystals. For example, Mn ions have been successfully incorporated into ZnSe and CdSe NCs. ${ }^{9,11}$ In heavily doped semiconductor $\mathrm{NCs}$, however, whether all doped Mn ions act as magnetic and optical impurities is not clear. Very recently, it was reported that an increase in the Mn concentration in single NCs leads to interactions among the $\mathrm{Mn}$ ions, which dramatically changes the luminescence properties. ${ }^{16}$ The magnetic circular dichroism (MCD) spectroscopy provides an opportunity to study electronic structures of $\mathrm{Mn}$ impurities and evaluate the Mn concentration in optically active NCs. ${ }^{9,17}$

In this letter, we report the magneto-optical properties of Mn-doped CdS NCs as a function of the Mn concentration by means of MCD spectroscopy. We find a strong correlation between the MCD and photoluminescence (PL) intensities in Mn-doped CdS NCs. At high concentrations (above 2-3 $\mathrm{mol} \% \mathrm{Mn}$ ions), antiferromagnetic $\mathrm{Mn}-\mathrm{Mn}$ pairs are formed

\footnotetext{
${ }^{\text {a) }}$ Author to whom correspondence should be addressed. Electronic mail: kanemitu@scl.kyoto-u.ac.jp.
}

in NCs, and they affect the magneto-optical and luminescence properties.

The reverse micelle method ${ }^{18-20}$ was used to fabricate Mn-doped CdS NCs with a ZnS shell layer (CdS:Mn/ZnS core-shell NCs) at varying Mn concentrations. The fabrication procedure and characterization were described in detail in Refs. 16 and 20. The molar ratio of $\mathrm{Mn}$ ions to $\mathrm{Cd}$ ions in the reverse micelle solutions was used for the Mn concentration in synthesized NCs. MCD spectra were obtained using a JASCO J-820 circular dichroism spectropolarimeter.

Figure 1 shows the optical absorption, PL, and MCD spectra of CdS:Mn/ZnS core-shell NCs with the Mn concentration of $2 \mathrm{~mol} \%$. All spectra are measured at room temperature. The lowest exciton band appears at around $2.9 \mathrm{eV}$ in the absorption spectrum. From the peak position and the calculation based on an effective mass approximation, the average core radius of the CdS:Mn/ZnS NCs is estimated to be about $2.0 \mathrm{~nm}$. The absorption spectra with different concentrations of Mn ions showed that the energy of the lowest excitons is almost independent of the Mn-doping concentrations. Therefore, no significant size change occurs by the doping of Mn ions into NCs. In the PL spectrum, the broad PL band appears at around $2.1 \mathrm{eV}$ and is assigned to the intra- $3 d$ transition of the Mn ions $\left({ }^{4} T_{1}-{ }^{6} A_{1}\right) .{ }^{5}$ Moreover, the broad MCD spectrum appears at the lowest exciton energy. The MCD peak signal is assigned to Zeeman splitting of the

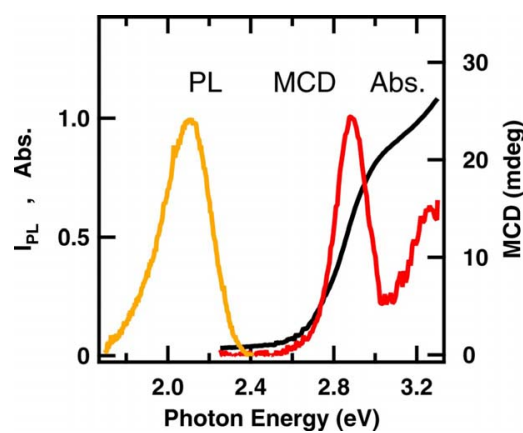

FIG. 1. (Color online) Optical absorption, PL, and MCD spectra of the Mn 2 mol \% doped CdS NCs. 


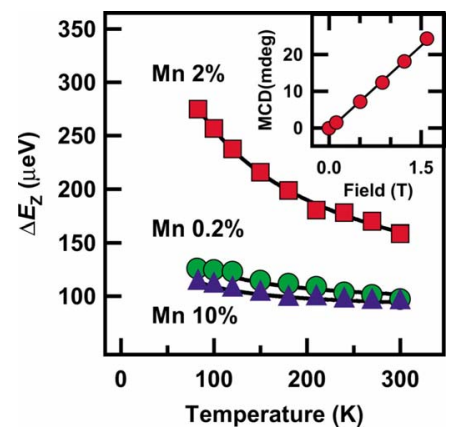

FIG. 2. (Color online) (a) Temperature dependence of the Zeeman splitting energy of CdS NCs doped with $0.2,2$, and $10 \mathrm{~mol} \% \mathrm{Mn}$ ions. The inset shows the magnetic field dependence of the MCD signal intensity in 2 mol \% Mn doped CdS NCs.

lowest exciton. The MCD signal is proportional to the Zeeman splitting and the first derivative of the absorption spectrum, where the Zeeman splitting is smaller than the bandwidth of the lowest exciton absorption. ${ }^{21}$ Here, we confirm that the spectral shape of the MCD signal is almost the same as the first derivative of the absorption spectrum. Therefore, we evaluated the Zeeman splitting $\Delta E_{Z}$ from the maximum values of the MCD spectrum (MCD) ${ }_{\max }$, and the first derivatives of the absorption spectrum $(d \alpha / d E)_{\max }$.

Figure 2 shows the temperature dependence of the Zeeman splitting of the $0.2,2$, and $10 \mathrm{~mol} \% \mathrm{CdS}: \mathrm{Mn} / \mathrm{ZnS}$ coreshell NCs. The 2 mol \% Mn-doped NCs show clear temperature dependence. Even in undoped CdS NCs, the Zeeman splitting occurs due to interaction between electron spins and applied magnetic field, and the Zeeman splitting of undoped NCs scarcely depends on temperature. Therefore, the temperature dependence of CdS:Mn/ZnS NCs is explained in the model by including the Zeeman splitting due to host $\mathrm{CdS}$ NCs, $\Delta E_{\mathrm{CdS}}$. In Mn-doped NCs, $s p-d$ exchange interactions cause additional splitting along with the Mn localized spin $\Delta E_{s p-d}$. The Mn concentration dependence of the Zeeman splitting in doped CdS NCs $\Delta E_{Z}(x)$ is described as ${ }^{22}$

$$
\Delta E_{Z}(x)=\Delta E_{\mathrm{CdS}}+\Delta E_{s p-d}(x)=g_{\mathrm{eff}} \mu_{B} B-N_{0}(\alpha-\beta) x\left\langle S_{z}\right\rangle,
$$

where $g_{\text {eff }}, N_{0} \alpha(\beta), x$, and $\left\langle S_{z}\right\rangle$ are the effective $g$-factor, the exchange integral between $\mathrm{Mn} d$-electrons and conduction band electrons (valence band holes), the mole fraction of $\mathrm{Mn}$ ions, and the averaged spin along the optical axis, respectively.

Since the splitting energy due to $s p-d$ exchange $\Delta E_{s p-d}$ is proportional to $\left\langle S_{z}\right\rangle$, which indicates $\mathrm{Mn}$ magnetization, $\Delta E_{s p-d}$ varies in accordance with the Curie law in paramagnetic Mn-doped NCs. The inset of Fig. 2 shows the peak intensity of the MCD signal as a function of the applied magnetic field. The linear field dependence of the MCD signal also indicates that our Mn-doped CdS NCs are paramagnetic. Therefore, the temperature dependence of the Zeeman splitting energy caused by $s p-d$ exchange, $\Delta E_{Z}(T)$, is described as

$$
\Delta E_{Z}(T)=\Delta E_{\mathrm{CdS}}+\Delta E_{s p-d}(T)=\Delta E_{\mathrm{CdS}}+\frac{C}{T},
$$

where $C$ is proportional to a Curie constant. The observed splitting energy can be fitted to Eq. (2). The obtained value

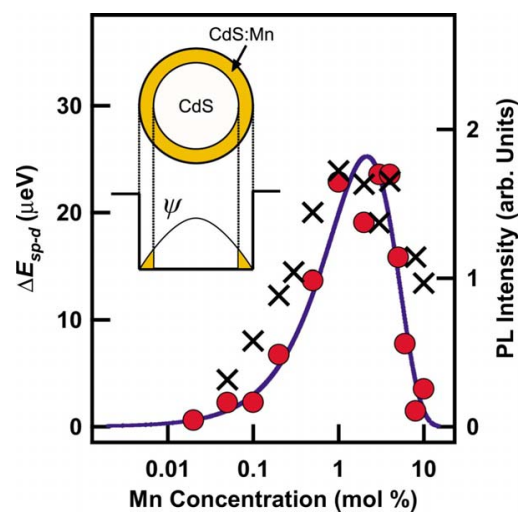

FIG. 3. (Color online) The splitting energy due to $s p-d$ exchange (circles) and PL intensities (crosses) of Mn-doped CdS NCs as a function of the Mn concentration. The PL data are taken from Ref. 16. The solid curve shows the calculated $s p$ - $d$ exchange energies using Eq. (3). Inset: Schematic illustration of model Mn-doped CdS NC. $\psi$ is the envelope function of electrons and holes.

of $\Delta E_{\mathrm{CdS}} \approx 120 \mu \mathrm{eV}$ in $2 \mathrm{~mol} \%$ doped NCs is in reasonable agreement with the splitting energy of undoped NCs. This agreement indicates that the splitting energy $\Delta E_{\mathrm{CdS}}$ is almost independent of the Mn concentration. The 2 mol \% doped $\mathrm{NCs}$ show larger temperature dependence than $0.2 \mathrm{~mol} \%$ doped NCs. The temperature-dependent splitting energy $\Delta E_{s p-d}$ increases with the number of $\mathrm{Mn}$ ions in concentrations below $2 \mathrm{~mol} \%$. More heavily doped (10 mol \%) NCs, however, show a weaker temperature dependence. The smaller value of $C$ indicates that $s p-d$ exchange coupling becomes weaker or that the number of magneto-optically active $\mathrm{Mn}$ ions with isolated spin decreases in heavily doped NCs.

To clarify the Mn concentration dependence of $s p-d$ exchange coupling, the Zeeman splitting energy under the applied magnetic field of $1.6 \mathrm{~T}$ was measured at room temperature. We extracted $\Delta E_{s p-d}$ by subtracting $\Delta E_{\mathrm{CdS}}$ from total Zeeman splitting energy. We used the splitting energy of undoped NC samples as $\Delta E_{\mathrm{CdS}}$ because we concluded that $\Delta E_{\mathrm{CdS}}$ was independent of $\mathrm{Mn}$ concentration, as described above. Note that the splitting energy of undoped CdS NC samples is about $107 \mu \mathrm{eV}$, which is larger than that of bulk crystals (cf., $88 \mu \mathrm{eV}$ at $1.6 \mathrm{~T}$ ). ${ }^{23}$ Similar differences between the NCs and bulk crystals have been reported in semiconductor NCs. ${ }^{24,25}$

Figure 3 summarizes the Mn-related splitting energy $\Delta E_{s p-d}$ at room temperature and as a function of the $\mathrm{Mn}$ concentration in NCs. At low Mn concentrations, $\Delta E_{s p-d}$ increases monotonically up to about $2-3 \mathrm{~mol} \%$ of $\mathrm{Mn}$ ions, and starts to decrease rapidly around $3 \mathrm{~mol} \%$. The decrease in $\Delta E_{s p-d}$ at high concentration cannot be explained by the saturation of impurity dopants, but suggests that many $\mathrm{Mn}$ ions are doped in $\mathrm{CdS} / \mathrm{ZnS}$ core-shell NCs. For comparison with PL properties, the Mn-related PL intensity is also plotted as a function of the Mn concentration. The data are taken from Ref. 16. A clear correlation between $\Delta E_{s p-d}$ and the PL intensity is observed. These behaviors indicate that in heavily doped NCs, Mn-Mn interactions play an important role in reducing the MCD and PL intensities. We also characterized some samples by electron spin resonant (ESR) spectroscopy and microwave induced plasma mass spectrometry (MIP-MS). In x-band ESR spectrum, hyperfine structures due to $\mathrm{Mn}^{2+}$ ions incorporated in a CdS host crys- 
tal were clearly observed in the 0.1 and $1 \mathrm{~mol} \%$ sample. ${ }^{16}$ In the MIP-MS experiments, the $\mathrm{Mn}^{2+}$ concentration of synthesized NCs was evaluated to be about $1.98 \mathrm{~mol} \%$ in the case of the initial $\mathrm{Mn}^{2+}$ concentration in solutions of $2 \mathrm{~mol} \%$. These results show that in lightly doped samples below 2-3 mol \%, Mn ions are doped in NCs. We consider that in heavily doped samples, almost Mn ions are located near the surface of the core and that the nearest neighbor Mn-Mn pair formation is a possible candidate for the reduction in the magnetic and luminescent activities. The antiferromagnetic coupling of Mn spins reduces the Mn magnetization and causes a quenching of both magneto-optic and luminescent activities.

To study the mechanism of Mn-Mn coupling more quantitatively, we considered the dependence of the magneto-optic signal to $\mathrm{Mn}$ concentration in Mn-doped CdS NCs. Considering the interface roughness and the NC shape, we use a three-dimensional lattice model including a surface accumulation effect. Indeed, the ideal two-dimensional array model cannot reproduce the experimental results. ${ }^{16}$ Here, we assume that the $\mathrm{Mn}$ ions are randomly distributed over the three-dimensional lattice of NCs (12 equivalent nearest neighbor cation sites). ${ }^{26}$ Thus, the probability $P_{i}(x)$ that a Mn ion is isolated from other Mn ions is described as $P_{i}(x)=(1$ $-x)^{12}$. Then the density of isolated Mn ions without antiferromagnetic coupling is given by $x(1-x)^{12}$. In addition, since the observed concentration dependence of the splitting cannot be explained by the simple picture that all $\mathrm{Mn}$ ions are homogeneously distributed over the $\mathrm{CdS}$ core, we assume that a large number of $\mathrm{Mn}$ ions are located near the interface between the core and the shell layer (in the near-surface region of the $\mathrm{CdS}$ core), as illustrated in the inset of Fig. 3. This accumulation increases the $\mathrm{Mn}$ concentration and the probability of $\mathrm{Mn}-\mathrm{Mn}$ antiferromagnetic pair formation. The $s p$ - $d$ exchange energy is given by

$$
\Delta E_{s p-d}(x)=A x\left(1-\frac{x}{c_{0}}\right)^{12}, \quad A=-N_{0}(\alpha-\beta)\left\langle S_{z}\right\rangle,
$$

where $c_{0}$ is the volume ratio of the accumulated near-surface region to the $\mathrm{CdS}$ NC. The solid line in Fig. 3 is a calculated result using Eq. (3). The observed data are well reproduced by our model with $c_{0}=0.3$ and $A=3.1 \mathrm{meV}$. This indicates that the doped $\mathrm{Mn}$ ions become $\mathrm{Mn}-\mathrm{Mn}$ pairs in heavily doped NCs and the pairs reduce the magneto-optic activity. Our findings also indicate that doped Mn ions accumulate in about $30 \%$ of the volume near the core-shell interface. This result indicates that most of the doped ions exist at the near edge region of the $\mathrm{CdS}$ core.

Note that the coefficient $A$ reflects the interaction between carriers and localized spins in Mn-doped CdS NCs and depends on the spatial distribution of isolated Mn ions. However, based on the calculation using the exchange integrals of the bulk crystal, ${ }^{22}$ the coefficient $A$ is estimated to be about $42 \mathrm{meV}$ at $1.6 \mathrm{~T}$ and $300 \mathrm{~K}$, and is larger than the experimental values. This discrepancy also indicates an inhomogeneous distribution of Mn ions in NCs and/or the surface accumulation of $\mathrm{Mn}$ ions, leading to the reduction in overlap between the wave functions of electrons (holes) and the doped Mn ions. Considering the overlap between the $1 S$-envelope function in the infinite spherical confinement potential and doped $\mathrm{Mn}$ ions and that $\mathrm{Mn}$ ions are distributed homogeneously in about $30 \%$ of the volume near the edge of the CdS core (see the inset of Fig. 3), we obtained $A$ $\sim 1.3 \mathrm{meV}$. The calculated result can explain the coefficient $A$ obtained by MCD experiments. Both the Mn concentration dependence of the Zeeman splitting and the magnitude of the obtained Zeeman splitting are explained by our model with an accumulation of the $\mathrm{Mn}$ ions to $30 \%$ volume near the edge of the CdS core. This also indicates that Mn impurities form pairs and are incorporated within NCs but located at the near-surface region of the $\mathrm{CdS}$ core in heavily doped $\mathrm{CdS} /$ $\mathrm{ZnS}$ core-shell NCs.

In conclusion, we report the dependence of the $s p-d$ exchange energy to the Mn concentration in Mn-doped CdS NCs and we find that in heavily Mn-doped samples, the antiferromagnetic coupling of Mn ion pairs causes a reduction in the magneto-optic signal intensity. The optimum concentration for luminescence is almost the same as for magnetooptical responses. Our findings provide information that is useful in the design of magneto-optical materials based on semiconductor NCs.

Part of this work was supported by KAKENHI (Contract Nos. 19740183 and 20104006).

${ }^{1}$ L. Brus, J. Phys. Chem. 90, 2555 (1986)

${ }^{2}$ Y. Kanemitsu, Phys. Rep. 263, 1 (1995).

${ }^{3}$ A. P. Alivisatos, J. Phys. Chem. 100, 13226 (1996).

${ }^{4}$ D. J. Norris, Al. L. Efros, and S. C. Erwin, Science 319, 1776 (2008).

${ }^{5}$ R. Beaulac, P. I. Archer, S. T. Ochsenbein, and D. R. Gamelin, Adv. Funct. Mater. 18, 3873 (2008).

${ }^{6}$ R. N. Bhargava, D. Gallagher, X. Hong, and A. Nurmikko, Phys. Rev. Lett. 72, 416 (1994).

${ }^{7}$ G. Counio, T. Gacoin, and J. P. Boilot, J. Phys. Chem. B 102, 5257 (1998).

${ }^{8}$ F. V. Mikulec, M. Kuno, M. Bennati, D. A. Hall, R. G. Griffin, and M. G. Bawendi, J. Am. Chem. Soc. 122, 2532 (2000).

${ }^{9}$ D. J. Norris, N. Yao, F. T. Charnock, and T. A. Kennedy, Nano Lett. 1, 3 (2001).

${ }^{10}$ Y. Kanemitsu, H. Matsubara, and C. W. White, Appl. Phys. Lett. 81, 535 (2002).

${ }^{11}$ S. C. Erwin, L. Zu, M. I. Haftel, Al. L. Efros, T. A. Kennedy, and D. J. Norris, Nature (London) 436, 91 (2005).

${ }^{12}$ A. Ishizumi, K. Matsuda, T. Saiki, C. W. White, and Y. Kanemitsu, Appl. Phys. Lett. 87, 133104 (2005).

${ }^{13}$ G. M. Dalpian and J. R. Chelikowsky, Phys. Rev. Lett. 96, 226802 (2006).

${ }^{14}$ Y. Yang, O. Chen, A. Angerhofer, and Y. C. Cao, J. Am. Chem. Soc. 128, 12428 (2006).

${ }^{15}$ D. A. Bussian, S. A. Crooker, M. Yin, M. Brynda, Al. L. Efros, and V. I. Klimov, Nature Mater. 8, 35 (2009).

${ }^{16}$ A. Ishizumi, E. Jojima, A. Yamamoto, and Y. Kanemitsu, J. Phys. Soc. Jpn. 77, 053705 (2008)

${ }^{17}$ D. M. Hoffman, B. K. Meyer, A. I. Ekimov, I. A. Merkulov, Al. L. Efros, M. Rosen, G. Couino, T. Gacoin, and J. P. Boilot, Solid State Commun. 114, 547 (2000).

${ }^{18}$ M. L. Steigerwald, A. P. Alivisatos, J. M. Gibson, T. D. Harris, R. Kortan, A. J. Muller, A. M. Thayer, T. M. Duncan, D. C. Douglass, and L. E. Brus, J. Am. Chem. Soc. 110, 3046 (1988).

${ }^{19}$ H. Yang, P. H. Holloway, G. Cunningham, and K. S. Schanze, J. Chem. Phys. 121, 10233 (2004).

${ }^{20}$ A. Ishizumi and Y. Kanemitsu, Adv. Mater. (Weinheim, Ger.) 18, 1083 (2006).

${ }^{21}$ K. Ando, K. Takahashi, T. Okuda, and M. Umehara, Phys. Rev. B 46, 12289 (1992).

${ }^{22}$ J. K. Furdyna, J. Appl. Phys. 64, R29 (1988).

${ }^{23}$ G. Blattner, G. Kurtze, G. Schmieder, and C. Klingshirn, Phys. Rev. B 25 , 7413 (1982)

${ }^{24}$ M. Kuno, M. Nirmal, M. G. Bawendi, A. Efros, and M. Rosen, J. Chem. Phys. 108, 4242 (1998).

${ }^{25}$ Y. Toda, S. Shinomori, K. Suzuki, and Y. Arakawa, Phys. Rev. B 58, R10147 (1998).

${ }^{26}$ D. S. McClure, J. Chem. Phys. 39, 2850 (1963). 